\title{
INSIDE LAB INVEST
}

PERIBILIARY FIBROGENIC CELLS-MODULATORS OF HEPATIC FIBROSIS: Hepatic fibrosis is thought to be caused in part by the activation of and subsequent extracellular matrix production and deposition by hepatic stellate cells. However, the presence of other mesenchymal cell populations, including portal fibroblasts, in the liver raises the possibility that several cell populations may, in aggregate, be responsible for hepatic fibrosis resulting from a variety of stimuli in a time- and location-specific manner. In this issue, using a rat model of biliary injury, specifically a double ligation and section of the common bile duct, Kinnman et al demonstrate the derivation of myofibroblastic cells from portal fibrocytes (Lab Invest 2003, 83: 163-173). The authors provide new data on the phenotypic differences between portal-derived and the hepatic stellate cell (HSC) sinusoidal-derived myofibroblasts. While the authors noted several similarities between HSC- and portal-derived myofibroblasts, including expression of fibulin 2, P100, and cellular retinal-binding protein-1, they noted a significantly lower production in IL-6 by the portal-derived myofibroblasts compared with HSC-derived myofibroblasts. Given the important pleiotropic roles of cytokines in the response to hepatic injury and subsequent repair, the differentiation of distinct myofibroblast populations (in this case from portal fibroblasts and HSC sinusoids) in specific temporal and spatial sequences are likely to have a significant impact upon the extent of regeneration and scarring following injury.

NEW MICROSCOPES ENTER THE SCENE: The availability of high throughput technologies to analyze tissues will bring about change in the way we diagnose, classify, treat, and prognosticate disease. In fact, the ensemble of these technologies can be thought of as new microscopes, instruments that enable us to describe diseases at the molecular level. In this issue, de Vos and collaborators provide us with data derived from an analysis of tumor progression in cases of follicular lymphoma (FL) (Lab Invest 2003, 83: 271-285). The transition of follicular tumors to diffuse large B cell lymphoma (DLBCL) has been well characterized from many view points, but to date there is still a paucity of comprehensive data sets that address the progression from FL to DLBCL. The work by de Vos et al provides an example of how the discovery steps need to be followed by validation using more targeted approaches (eg, QRT-PCR and IHC).Because the progression pathways are likely to be heterogeneous, a large number of cases will be needed before we gain a systems overview of progression and before we can focus on specific genes as therapeutic targets or as predictors of response to therapeutic intervention. Thus, we can look forward to many studies that will follow this one. By now it is clear that adjuvant approaches to tissue analysis will greatly contribute to the personalized predictive medicine that will be practiced in much of the 21 st century. 